\title{
FORUM
}

\section{„Brügge sehen und sterben“ - Gemeinschaftsmethode versus Unionsmethode}

\author{
Manuel Sarrazin und Sven-Christian Kindler ${ }^{*}$
}

Es ist eine alte Faustregel: In Krisenzeiten steigt der Handlungsdruck und damit auch Bedeutung und Macht der Regierungen und Exekutiven. Wenn schnelle Entscheidungen zu komplexen und international auftretenden Krisen benötigt werden, empfinden es Abgeordnete und Öffentlichkeit als legitim, wenn die Exekutive ihren Handlungsspielraum schnell und weitgehend nutzt. In den letzten Jahren ist dieser Effekt auch in Bezug auf den politischen Umgang mit der Krise in der Euro-Zone zu beobachten. Dabei offenbart sich eine wesentliche Besonderheit: In dieser Krise hat auf europäischer Ebene vielmehr der Rat, insbesondere die Staats- und Regierungschefs der Mitgliedstaaten im Europäischen Rat, die Rolle der Entscheidungen treffenden Exekutive übernommen, als die nach einer klassischen Interpretation des europäischen Institutionengefüges am ehesten in die Rolle einer Exekutive passende Europäische Kommission. Mitunter haben der französische Präsident und die deutsche Bundeskanzlerin sogar den bewährten Interessenausgleich zwischen den Staaten im Europäischen Rat ausgehebelt, um, wie beim berühmten Strandspaziergang von Deauville, zu zweit zu versuchen, für Europa zu entscheiden. Daraus ergeben sich grundlegende Fragen für die Zukunft der Demokratie in Europa: Wie Bürgerinnen und Bürger bei der Krisenbewältigung beteiligt werden und mitentscheiden können, wie die Rechte von nationalen Parlamenten und des Europäischen Parlaments gewahrt werden. Die Entwicklung der letzten Jahre wollen wir im Folgenden beschreiben und analysieren: Welche Auswirkungen hat die Stärkung der Regierungen auf die Legitimität von politischen Entscheidungen in der Öffentlichkeit, das Verfahren ihrer demokratischen Legitimation, die Handlungsfähigkeit der Politik, die Effizienz des Krisenmanagements in der Europäischen Union und auf die künftige Integration?

Am 2. November 2010 hat Bundeskanzlerin Angela Merkel in ihrer Rede anlässlich der Eröffnung des 61. Akademischen Jahres des Europakollegs Brügge ${ }^{1}$ den Begriff der Unionsmethode und die ideologische Grundlage für eine Verschiebung der Entscheidungsstrukturen und Machtzentren in der europäischen Einigung eingeführt. Die Gemeinschaftsmethode sei „,natürlich eine Methode der europäischen Rechtsetzung“, 2 aber sie müsse gerade in der Krise und gerade außerhalb der Gemeinschaftskompetenzen im Sinne der Subsidiarität ergänzt werden durch die intergouvernementale Methode, die neue „Unionsmethode“. 3

* Manuel Sarrazin, MdB, Sprecher für Europapolitik und Obmann im Europaausschuss, Fraktion Bündnis 90/Die Grünen.

Sven-Christian Kindler, MdB, Mitglied im Haushaltsausschuss, Fraktion Bündnis 90/Die Grünen.

1 Rede von Bundeskanzlerin Merkel anlässlich der Eröffnung des 61. Akademischen Jahres des Europakollegs Brügge. Di, 2.11.2010 in Brügge, abrufbar unter: http://www.coleurope.eu/file/content/news/Speeches/Rede \%20Merkel\%20Europakolleg\%20Bruegge.pdf (letzter Zugriff: 29.6.2012).

2 Ebenda, S. 5.

3 Zur Unterscheidung der verschiedenen Methoden siehe auch Antonio Missiroli: Eine kleine Abhandlung über Methoden und ihre Formen in der EU-Praxis, in: integration 3/2011, S. 247-256. 
Merkels Unionsmethode hat in den letzten Jahren wichtige Aspekte des Handelns der Europäischen Union in der Krise in der Euro-Zone bestimmt. Keineswegs haben die Staatsund Regierungschefs aber nur dann auf sie zurückgegriffen, wenn ein Kompetenz- oder Kapazitätsmangel - wie beispielsweise bei der Einrichtung der Europäischen Finanzstabilisierungsfazilität (EFSF) - ein Handeln der Gemeinschaftsinstitutionen unmöglich machte. Vielmehr kommt die Unionsmethode vielen Staats- und Regierungschefs sowie nationalen Politikerinnen und Politikern zu pass. Sie sichert ihnen Macht und Aufmerksamkeit in den nationalen europapolitischen Debatten. Es bleibt festzuhalten: In dem Moment als Merkel sich hinter die europäischen Institutionen hätte stellen müssen, hat sie diese geschwächt. Mit der Unionsmethode untergräbt der Europäische Rat fortwährend die Autorität der Gemeinschaftsinstitutionen, deren Stärke gebraucht wird um diese Krise zu lösen. In Anlehnung an den Kinofilm „Brügge sehen... und sterben“ könnte Merkels Unionsmethode eine existenzielle Gefahr für die künftige europäische Integration darstellen. ${ }^{4}$

\section{Brügge sehen...: Die ,Unionsmethode ${ }^{6}$ in der politischen Realität}

Mit dem Inkrafttreten des Vertrags von Lissabon am 1. Dezember 2009 wurden die aufgrund der Beschlüsse der Gipfelkonferenz von Paris seit 1975 mindestens zweimal im Jahr stattfindenden Treffen der Staats- und Regierungschefs - für die sich der Begriff Europäischer Rat entwickelt hatte - als Organ der Europäischen Union anerkannt. ${ }^{5}$ Zwar kann der Europäische Rat auch heute keine Gesetzgebungsakte beschließen, aber die Verträge geben ihm doch eine Beschlusskompetenz über Rechtsakte und die Zusammensetzung verschiedener europäischer Funktionen und Ämter, wie beispielsweise zur Ernennung des Präsidenten der Europäischen Kommission. ${ }^{6}$ Der Vertrag von Lissabon hat dennoch insgesamt seine politische Vormachtstellung im Institutionengefüge der Europäischen Union untermauert.

Dem Wunsch nach der starken Legitimation wichtiger Entscheidungen auf europäischer Ebene gegenüber den Kabinetten und Parlamenten in den Mitgliedstaaten wurde zudem dadurch Rechnung getragen, dass im Europäischen Rat seit 2009 die Rolle der Staats- und Regierungschefs gestärkt wurde, so nehmen gemäß Art. 15 Abs. 2 und 3 Vertrag über die Europäische Union (EUV) die Außen- beziehungsweise Europaminister nur noch teil, wenn der Europäische Rat dies beschließt.

Besonders bedeutsam ist das in Deutschland. Zwar verantwortet das Auswärtige Amt offiziell weiterhin die Europakoordination innerhalb der Bundesregierung und die Vorbereitung der Tagungen des Europäischen Rates. In der Praxis spielt das Auswärtige Amt hier aber nur noch eine untergeordnete Rolle. Gerade der Europäische Rat und die informellen und bilateralen Gipfeltreffen haben aber in der Krise eine immer entscheidendere Bedeutung erlangt. Das zeigen deutlich die folgenden Entwicklungen: Durch die intergouvernementale Struktur der wesentlichen Teile der sogenannten Rettungsschirme ist die Ausgestaltung der damit verbundenen Ausweitung der europäischen Fähigkeiten in die Verantwortung der Finanzministerinnen und Finanzminister der Euro-Gruppe gelegt worden. Politisch entscheidend waren beispielsweise beim Europäischen Stabilitätsmechanismus (ESM) eben nicht die

4 Brügge sehen... und sterben, Regie Martin McDonagh, Tobis Film GmbH \& Co KG, 2008.

5 Vgl. Reiner Schulze/Manfred Zuleeg/Stefan Kaddelbach (Hrsg.): Europarecht. Handbuch für die deutsche Rechtspraxis, 2. Auflage, Baden-Baden 2010, S. 55.

6 Als Übersicht über den Kompetenzkatalog siehe Jean-Claude Piris: The Lisbon Treaty. A Legal and Political Analysis, Cambridge 2010, Anhang 6, S. 379-382. 
Debatten über die Ergänzung der Verträge auf dem Wege einer einfachen Vertragsänderung in Art. 136 Vertrag über die Arbeitsweise der Europäischen Union (AEUV), sondern die Ausgestaltung des völkerrechtlichen Vertrags zur Gründung des ESM. Hier führten die Finanzministerien die Debatten über Verfahren, Konditionalitäten, Stimmrechte, Ausleihvolumen und Beteiligung des privaten Sektors. Selbige sind folgerichtig auch die entscheidenden tagespolitischen Akteure in den Gremien der EFSF und des künftigen ESM. Hier entscheidet der identische Personenkreis der Euro-Gruppe über Schicksalsfragen der Europäischen Union. Auch aus Sicht der Öffentlichkeit haben neben den Regierungschefs die Finanzministerien die größte Verantwortung. ${ }^{7}$ Auf der anderen Seite entstehen zunehmend Strukturen einer wirtschaftspolitischen Steuerung in der Europäischen Union, die gemeinsam mit den Entscheidungen des Europäischen Rates faktisch die Rolle einer künftigen Wirtschaftsregierung übernehmen können. Alles in allem kann man der Euro-Gruppe attestieren, strukturell in der Krise zu einem der entscheidenden Gremien geworden und heute sehr viel bedeutender zu sein, als es die Verträge vorsehen.

Das gilt auch für den Wirtschafts- und Finanzausschuss (WFA) beim Rat der Europäischen Union, der künftig eine Schlüsselfunktion für Fragen in der Euro-Krise und der wirtschaftspolitischen Steuerung entwickeln könnte und gleichzeitig faktisch fast komplett außerhalb parlamentarischer Kontrolle steht. ${ }^{8}$

Vor dem Hintergrund dieser Veränderungen in der Gremien- und Entscheidungsstruktur lässt sich eine integrationspolitische Schwäche der klassischen Europaministerien und insbesondere des Auswärtigen Amtes als Europaressort innerhalb der Bundesregierung attestieren. Die Eigenart der immer häufiger tagenden informellen und offiziellen Gipfel hat zudem die Staats- und Regierungschefs mehr als früher in die Position versetzt, Verhandlungsprozesse in der Krise weitgehend selbst mit ihren Stäben zu begleiten oder im Hauptstadtformat von den Finanzministerien vorbereiten zu lassen. Bilaterale Gipfeltreffen und Initiativen, insbesondere von Deutschland und Frankreich, haben die Rolle der üblichen Ratsstrukturen und des Ausschusses der Ständigen Vertreter geschwächt. In die abschließende Entscheidungsfindung in den letzten Tagen vor einem Gipfel und auf dem Gipfel sind oftmals die weiteren Fachressorts und das Auswärtige Amt kaum einbezogen. Wie eigenmächtig gerade das Kanzleramt agiert, um der Kanzlerin eine dominierende Rolle in der Berichterstattung zu sichern, zeigte insbesondere der deutsch-französische Vorschlag für einen Pakt für Wettbewerbsfähigkeit, der auch ohne eine Einigung innerhalb der Bundesregierung als Initiative im Europäischen Rat eingebracht wurde. ${ }^{9}$

Gerade in der Situation der Krise hätte ein stärkeres und rascheres integrationspolitisches Engagement für eine tiefere europäische Integration und für strukturelle Veränderungen in den Verträgen das Europaressort innerhalb der Bundesregierung stärken können. Die gilt besonders mit Blick auf das Ziel, die sich entwickelnden neuen Verfahren und Strukturen möglichst eng an die Gemeinschaftsinstitutionen und die eingespielten innerstaatlichen Verfahren der Beteiligung von Bundestag, Bundesländern und Regierungsressorts zu binden.

7 So beschlossen die Staats- und Regierungschefs der Euro-Zone über die europäisch koordinierten bilateralen Finanzhilfen für Griechenland. Zwar entsprach die Ratserklärung nur einer politischen Vereinbarung, aber die politisch maßgebende Entscheidung fiel eben in diesem Format der Euro-Gruppe der Staats- und Regierungschefs. Vgl. Erklärung der Staats- und Regierungschefs des Euro-Währungsgebietes, Brüssel, den 7. Mai 2012.

8 Vgl. § 5 Abs. 4 des Gesetzes über die Zusammenarbeit von Bundesregierung und Deutschem Bundestag in Angelegenheiten der Europäischen Union (EUZBBG).

9 Das geht aus einem internen Arbeitspapier der Bundesregierung hervor. Vgl. Pakt für Wettbewerbsfähigkeit. Gesamtvorbehalt von BMWi und AA, abrufbar unter: http://www.euractiv.de/fileadmin/images/Pakt_Wettbewerbsfaehigkeit.pdf (letzter Zugriff: 29.6.2012). 
Dass das Auswärtige Amt gerade auf diesem Feld viel zu lange nicht vorgekommen ist, ist wesentlich der Passivität des Außenministers zuzuschreiben.

Seinen vorläufigen Höhepunkt erreicht das Prinzip der Unionsmethode mit den Vereinbarungen des Euro-Gipfels vom 8. November 2011, der neben der Festlegung, mindestens zweimal jährlich als Euro-Gipfel zu tagen, ganz ausdrücklich den Anspruch formuliert „,weiterhin eine zentrale Bedeutung beim täglichen Management des Euro-Währungsgebiets“ zu haben, „eine zentrale Rolle bei der Durchführung des Europäischen Semesters durch die Mitgliedstaaten des Euro-Währungsgebiets“ zu spielen und dazu eine eigene ständige Präsidentschaft einzurichten. ${ }^{10}$ Zur besseren Vorbereitung dieser Sitzungen ist bereits das Sekretariat des Wirtschafts- und Finanzausschusses/der Eurogruppen-Arbeitsgruppe mit sechs Stellen verstärkt worden. Immerhin sind diese Stellen sowie die bis zu zwei Stellen für den Vollzeitvorsitzenden der Eurogruppen-Arbeitsgruppe innerhalb und nicht außerhalb der Strukturen des Rates der Europäischen Union beziehungsweise der Kommission angesiedelt. ${ }^{11}$ Der Vertrag über Stabilität, Koordinierung und Steuerung in der Wirtschafts- und Währungsunion (Fiskalvertrag) kann eine völkerrechtliche Verewigung des Euro-Gipfels bedeuten, wenn das in Art. 16 des Vertrags festgelegte Ziel der Überführung der Vertragsinhalte in das Gemeinschaftsrecht nicht gelingen sollte. Die bisherigen Aussagen der britischen Regierung lassen nicht darauf schließen, dass der Fiskalvertrag innerhalb von fünf Jahren in Unionsrecht überführt werden kann. Es besteht die realistische Gefahr einer dauerhaften Parallelstruktur durch den Fiskalvertrag.

Festzuhalten ist: Die von Merkel in Brügge propagierte Unionsmethode hat die Verfahren und Strukturen der Europäischen Union verändert. Dabei stärkt sie die Macht der nationalen Regierungen, insbesondere der Euro-Zone gegenüber der Europäischen Kommission und des nur in Teilbereichen der Reform des Stabilitäts- und Wachstumspakts und der Finanzmarktregeln relevant involvierten Europäischen Parlaments. Die Aushandlungsprozesse im Europäischen Rat folgen dabei immer stärker dem von Merkel ausgegebenen Prinzip „Abgestimmtes solidarisches Handeln - jeder in seiner Zuständigkeit, alle für das gleiche Ziel." 12 So lange auch nur ein Mitgliedstaat das Ziel oder die Zuständigkeit infrage stellt, ist kein Fortschritt möglich. Das mit dem Vertrag von Lissabon gestärkte Prinzip der Mehrheitsentscheidung im Rat der Europäischen Union findet durch die Vormachtstellung der Unionsmethode im Rahmen der Maßnahmen zur Bewältigung der Krise in der Euro-Zone kaum Anwendung.

Dass diese Stärkung und partielle Wiedereinführung des Konsensprinzips gescheitert ist, zeigte sich spätestens an der Blockade der Initiative zur Vertragsänderung im Rahmen des Dezembergipfels 2011 durch das Vereinigte Königreich. Schließlich verband die Regierung Cameron die Frage der Ergänzung der Euro-Protokolle der EU-Verträge mit Fragen der Finanzmarktregulierung. Mit dem daraufhin vereinbarten Fiskalvertrag, an dem sich voraussichtlich 25 der 27 Mitgliedstaaten beteiligen werden, weicht Merkel letztlich selbst von dem in Brügge gepredigten Prinzip der Einigkeit ab. Sie schafft eine Struktur, die - im Widerspruch zu den Bekenntnissen von Brügge - eben nicht über die Anwendung der intergouvernementalen Methode „einen gemeinsamen europäischen Standpunkt schafft““ ${ }^{13}$

10 Vgl. Euro Summit Statement, Brussels, 26 October 2011.

11 Antwort des Parlamentarischen Staatssekretärs Steffen Kampeter vom 13. März 2012 auf eine schriftliche Frage des Abgeordneten Manuel Sarrazin, in: Schriftliche Fragen mit den in der Woche vom 12. März 2012 eingegangenen Antworten der Bundesregierung, Bundestagsdrucksache 17/9002, S. 17.

12 Rede von Bundeskanzlerin Merkel, 2010, S. 6-7.

13 Ebenda. 
Vielmehr droht, dass die Unionsmethode perspektivisch auch in die Zuständigkeiten der Gemeinschaftsinstitutionen Rat der Europäischen Union, Kommission und Europäisches Parlament eingreifen könnte und dort zum Teil eine Rückkehr zum Konsensprinzip bedeuten könnte. Das Scheitern der Unionsmethode „einen gemeinsamen europäischen Standpunkt“ zu schaffen, könnte also mit einer fortdauernden Schwächung der EU-Institutionen und des aquis einhergehen.

\section{„Brügge, wo ist denn das?“ - Die Handlung der Unionsmethode}

Waren die völkerrechtlichen Vereinbarungen der EFSF und des permanenten Euro-Rettungsschirms ESM unter Rückgriff auf die nationalen Budgets noch zumindest auch aus der Not der zu geringen finanziellen Kapazität des Gemeinschaftshaushalts geboren, so könnte der Fiskalvertrag - unabhängig von der konkreten ökonomischen Bewertung - der Ausgangspunkt eines neuen Prinzips der europäischen Integration sein. ${ }^{14}$ Für die aufgrund der Krise notwendigen strukturellen Veränderungen und Kompetenzverlagerungen könnte demnach eine neue völkerrechtliche Säule der europäischen Integration außerhalb der Organe und Institutionen der Europäischen Union entwickelt werden. Diese weitere europäische Integration könnte ausdrücklich neben und nicht unter oder innerhalb der Integration im Rahmen der Europäischen Union stehen. Dieses Szenario wurde beispielhaft vom Prozessvertreter der Bundesregierung Ulrich Häde im Organstreitverfahren der Bundestagsfraktion von Bündnis 90/Die Grünen gegen die Verletzung der Rechte des Bundestags aus Art. 23 Abs. 2 Grundgesetz (GG) durch die Bundesregierung vertreten. ${ }^{15}$

Die Unionsmethode wird vor allem seitens der Bundesregierung gegenüber der Öffentlichkeit mit der Legitimität der Euro-Rettungspolitik, der Bindung an die starke demokratische Legitimation der nationalstaatlichen Strukturen und dem Argument eines handlungsfähigen und effizienten Politikmanagements verteidigt. Unabhängig davon, dass ein entschiedeneres Handeln und eine von Anfang an nicht nur auf prozyklisches Sparen angelegte Euro-Rettungspolitik der Bundesregierung viele negative wirtschaftliche Auswirkungen hätte verhindern und damit die Legitimität der politischen Entscheidungen steigern können, ist auch die Methode der Euro-Rettungspolitik von entscheidender Bedeutung für ihre Legitimität. Dass in der Unionsmethode die stärkste Legitimität der Entscheidungen auf europäischer Ebene aufgrund der starken persönlichen Legitimität der Staats- und Regierungschefs erreicht werde, lässt sich nicht belegen. Eher gilt das Gegenteil, wie noch gezeigt werden wird. Zunächst ist festzuhalten, dass die bereits skizzierte Veränderung im Institutionengefüge insbesondere das Europäische Parlament als Vertretung der Unionsbürgerinnen und Unionsbürger an den Rand gedrängt hat. Die Entscheidung über das Wohl und Wehe der gemeinsamen Währung der Unionsbürgerinnen und Unionsbürger findet so plötzlich nicht mehr im von ihnen gewählten Europäischen Parlament, sondern vor allem in geheimen Sitzungen und Verhandlungen der Regierungen statt, über die oftmals kaum belastbare Informationen nach außen dringen. Das Wesen dieser Verhandlungen zeichnet sich insbesondere in der öffentlichen Debatte in Deutschland dadurch aus, dass in den meisten Fragen abseits der konkreten Kreditprogramme für Euro-Staaten auch der Deutsche Bundestag nicht

14 Es stand im Mai 2010 durchaus die Alternative einer Lösung innerhalb der Gemeinschaftsinstitutionen beispielsweise unter Anwendung der Flexibilitätsklausel im Raum. Die Einrichtung des Europäischen Finanzstabilisierungsmechanismus (ESFM) zeigt dieses deutlich.

15 Die Klage notiert unter dem Aktenzeichen Bundesverfassungsgericht 2 BvE 4/11. Vgl. auch das Urteil BVerfG, 2 BvE 4/11 vom 19.6.2012. 
ausreichenden Zugang zu Informationen oder Unterrichtungen hat. So fehlt letztlich auch dem Bundestag die Möglichkeit einer gut informierten Kommentierung der Politikentwicklung. Tatsächlich musste sich der Bundestag beispielsweise während der Verhandlungen über den ESM-Vertrag und den Euro-Plus-Pakt die Informationen auf allen möglichen Wegen - unter anderem durch freundliche Hilfe des österreichischen Parlaments - selbst beschaffen. Eine informierte Mitwirkung des Parlaments im Sinne des Art. 23 GG zu einem frühzeitigen und einflussreichen Zeitpunkt passte offenkundig nicht zur Eigenart der Regierungsverhandlungen der Unionsmethode. Die wiederholten Abstimmungen des Bundestags zur Ratifizierung der Euro-Rettungsschirme oder im Rahmen der erkämpften Beteiligungsrechte bei Einzelentscheidungen im Rahmen der Rettungsschirme (Kredithilfen, Hebelungen) schaffen sicherlich eine Legitimation. Allerdings ist diese Legitimation von Mitgliedstaat zu Mitgliedstaat sehr unterschiedlich und kann keinesfalls die Legitimation durch die aktive Beteiligung der Parlamente auch am Politikentwicklungsprozess ersetzen.

Die Beteiligung der nationalen Parlamente bedeutet, die Legitimation zu stärken, sie darf aber eben nicht zu einem Letztentscheidungsrecht reduziert werden, sondern muss zumindest in Deutschland dem Prinzip der informierten Mitwirkung an Gemeinschaftsverfahren entsprechen. Ausgerechnet bei diesen Entscheidungen über Milliardensummen sorgte die Unionsmethode in Deutschland für Mängel in der demokratischen Legitimation, die zum Urteilspruch des Bundesverfassungsgerichts auf Klage der Grünen Bundestagsfraktion führten, die Bundesregierung habe die Beteiligungsrechte des Bundestages aus Art. 23 Abs. 2 GG verletzt. Der Versuch der Bundesregierung, den ESM und den Euro-Plus-Pakt und damit auch alle weiteren intergouvernementalen Anbauten an die Europäische Union außerhalb des Art. 23 Abs. 2 GG und damit außerhalb der Mitwirkung von Bundestag und Bundesrat anzusiedeln, ist damit gescheitert. Das Bundesverfassungsgericht hat klargestellt, dass auch völkerrechtliche Verträge, ,,wenn sie in einem Ergänzungs- oder sonstigen besonderen Näheverhältnis zum Recht der Europäischen Union stehen“" zu den Angelegenheiten der Europäischen Union im Sinne von Art. 23 Abs. 2 GG gehören. ${ }^{16}$ Der Zweite Senat nutzte das Urteil zudem um qualitative und quantitative Standards der Unterrichtung des Bundestags und der Ermöglichung der Wahrnehmung seiner Mitwirkungsrechte festzuschreiben. ${ }^{17}$ In seinem Urteil betont das Bundesverfassungsgericht ausdrücklich die Bedeutung der öffentlichen parlamentarischen Beratung und Beschlussfassung auch von Angelegenheiten der Euro-Rettung vor Entscheidungen von ,erheblicher rechtlicher oder faktischer Bedeutung “. ${ }^{18}$ Gerade die aktive Beteiligung des Deutschen Bundestags und die öffentliche parlamentarische Willensbildung stärke gleichzeitig ,die Responsivität von europäischen Entscheidungen für die Interessen und Überzeugungen der Bürger". ${ }^{19}$ Die Rechte des Deutschen Bundestags (und des Bundesrats), die laut Bundesverfassungsgericht dem im Demokratieprinzip verankerten Grundsatz parlamentarischer Öffentlichkeit dienen, sind zumindest innerstaatlich nur im Rahmen der Verfahren gewährleistet, die für die Parlamente in der Gemeinschaftsmethode gelten. ${ }^{20}$ Das Gericht selber attestiert der Methode Merkel also ein verfassungswidriges Legitimationsdefizit durch den Versuch der Umgehung von Information und Mitwirkung der Parlamente. Mit seinem Urteil beendet das Bundesverfassungsgericht damit innerstaatlich faktisch die Praxis der Unionsmethode. Diese Bewertung der Unions-

16 BVerfG, 2 BvE 4/11 vom 19.6.2011, Leitsatz 1.

17 Ebenda, Zusammengefasst insbesondere in den Leitsätzen 2 und 3.

18 Ebenda, Rn. 114.

19 Ebenda, Rn. 113.

20 Ebenda, Rn. 112. 
methode und der Bedeutung der parlamentarischen und damit öffentlichen Debatte durch das Bundesverfassungsgericht sollte auch für die Verfahren auf europäischer Ebene und insbesondere die fehlenden Mitwirkungsrechte des Europäischen Parlaments zu denken geben.

Doch auch die parlamentarische Legitimation fast nur durch nationale Parlamentsentscheidungen kann weitgehende Legitimationskonflikte hervorrufen. Dies zeigte beispielsweise der Fall der Fast-Ablehnung der EFSF-Volleffektivierung im slowakischen Parlament, als in einem Mitgliedstaat eine einen relativ kleinen Wählerinnen- und Wähleranteil auf sich vereinigende Partei fast in die Lage versetzt worden wäre, ultimativ eine Politikentscheidung für die ganze Euro-Zone zu verhindern.

Zudem kann sie zwar Schwächen der Legitimation von Entscheidungen auf europäischer Ebene aufgrund der fehlenden Beteiligung des Europäischen Parlaments lindern, diese aber weder ausgleichen noch ersetzen, schon weil die entscheidende Rolle, gerade des Deutschen Bundestags, bei Schicksalsentscheidungen für andere Staaten sehr leicht als besonders illegitim aufgefasst werden kann. ${ }^{21}$ Perspektivisch wird nicht die Unionsmethode, sondern nur eine stärkere Einbindung des Europäischen Parlaments die Legitimation der Euro-Rettungspolitik steigern können, denn nur so wird für jede und jeden sich betroffen fühlenden Unionsbürger/in in gleichem Maße die Abwählbarkeit der Entscheidungsträger/innen sichergestellt werden können. Eine starke Rolle der nationalen Parlamente steht nicht in Konkurrenz dazu, sondern kann die Legitimation stärken.

Die Unionsmethode bringt noch eine weitere Schwächung der Legitimität der Euro-Rettungspolitik mit sich. Die klassischen Gipfelkonferenzen sind aufgrund ihrer großen Bedeutung für die nationalen Öffentlichkeiten davon geprägt, sich in der Berichterstattung besonders stark auf die Bewertung des persönlichen Erfolgs des beziehungsweise der jeweiligen Staats- oder Regierungschefs zuzuspitzen. Besonders in den schwierigen Fragen eines Ausgleichs der sehr divergenten Interessen der Mitgliedstaaten im Fall der Euro-Rettungspolitik ist aber Flexibilität und Kompromissfähigkeit der jeweiligen Positionen von Bedeutung. Gerade die schwarz-gelbe Koalition in Berlin hat nach einer sehr harten Betonung der eigenen Linie vor den Gipfelkonferenzen leider nur selten auch eine klare Kommunikation der erreichten Kompromisse nach außen vorgenommen. Vielmehr wurden die Gipfel praktisch unabhängig vom Ergebnis zum Nachweis der persönlichen Stärke der Kanzlerin hervorgehoben - eine Bewertung, die sich mit den real erreichten für alle Seiten schmerzhaften Kompromissen insbesondere in der Zeit bis zum Oktober 2011 kaum deckte, auch vor dem Hintergrund der Anfangs komplett ablehnenden deutschen Position gegenüber den Euro-Rettungsschirmen. Diese der Unionsmethode innewohnende Logik, dass die Staats- und Regierungschefs die erzielten Kompromisse auf den Gipfeln nur selten in angemessener Art nach Hause kommunizieren können und wollen, sondern immer als ,Gewinner/in' und ,Wahrer/innen der nationalen Interessen gegen die Anderen` auftreten müssen, hat dazu beigetragen, dass nationale Ressentiments gestärkt wurden und der Gedanke der europäischen Solidarität in den Hintergrund getreten ist. Dies verschleiert komplexe ökonomische Zusammenhänge der Finanzkrise in der Euro-Zone, zum Beispiel die Frage der Leistungsbilanzdefizite und -überschüsse, und reduziert die Krise fälschlicherweise auf Probleme nationaler Haushaltspolitik. Es ist also auch ein Ergebnis der Unionsmethode, dass in vielen

21 So hat beispielsweise die Veröffentlichung von Plänen der irischen Regierung für Steuererhöhungen in Irland aufgrund eines Lecks im Deutschen Bundestag einen handfesten Skandal in Irland ausgelöst. Vgl. SpiegelOnline: Irland empört sich über deutsches Datenleck, 18.11.2011. 
Mitgliedstaaten der Europäischen Union inzwischen ein Eindruck vorherrscht, eine anscheinend ökonomisch nachteilig wirkende ,aus Berlin diktierte' Krisenpolitik gar nicht mehr durch Wahlen im eigenen Land verändern zu können. Besonders deutlich wird das an den unterschiedlichen Debatten in Europa zur jeweiligen nationalen Umsetzung des Fiskalvertrags. So gilt der Vertrag als Mittel der Durchsetzung deutscher Stabilitätsinteressen. Dabei ist klar: Die bisherigen Regeln im Fiskalpakt reichen für eine solide und gerechte Haushaltspolitik nicht aus.

Die Unionsmethode sorgt also weder für eine stärkere öffentliche noch formale Legitimation der Entscheidungen, sondern schwächt diejenigen Akteure - insbesondere die Parlamente -, die die politische Legitimationskette bis hin zu den Bürgerinnen und Bürgern knüpfen können.

Auch die Handlungsfähigkeit und Entscheidungseffizienz erweist sich nur schwerlich als ein stichhaltiges Argument für die Nutzung der Unionsmethode. Bei der Finanzmarktregulierung, bei der Finanztransaktionssteuer, bei der Verschärfung der Regeln und Verfahren des Stabilitäts- und Wachstumspaktes, bei der Steuerkooperation und der Bekämpfung von Steuerhinterziehung oder in der Debatte um Eurobonds und einen europäischen Altschuldentilgungsfonds gilt: Viele der entscheidenden Fortschritte bei der Bekämpfung der Krise sind vor allem von den Gemeinschaftsinstitutionen eingefordert oder vorangetrieben worden, während der Rat der Europäischen Union durch das Wiedererstarken des Veto-Spiels nur schwer in der Lage ist, rasche Aushandlungen zwischen den unterschiedlichen nationalstaatlichen Interessen vorzunehmen und sich oftmals auf materiell eher symbolische Politikvereinbarungen wie beim Euro-Plus-Pakt oder beim Fiskalpakt zurückzieht. Auf dem ,Fahrersitz' bei der Entwicklung des Europäischen Semesters, der makroökonomischen Überwachung und Steuerung und der Reform des Stabilitäts- und Wachstumspaktes ${ }^{22}$ sind sehr klar die Europäische Kommission und in Teilen auch das Europäische Parlament zu finden. ${ }^{23}$ Auch bei den Debatten über Projektbonds und Eurobonds sind Europäische Kom-

22 Vgl. exemplarisch das sogenannte, six pack‘ bestehend aus Verordnung (EU) Nr. 1173/2011 des Europäischen Parlaments und des Rates vom 16. November 2011 über die wirksame Durchsetzung der haushaltspolitischen Überwachung im Euro-Währungsgebiet; Verordnung (EU) Nr. 1174/2011 des Europäischen Parlaments und des Rates vom 16. November 2011 über Durchsetzungsmaßnahmen zur Korrektur übermäßiger makroökonomischer Ungleichgewichte im Euro-Währungsgebiet; Verordnung (EU) Nr. 1175/2011 des Europäischen Parlaments und des Rates vom 16. November 2011 zur Änderung der Verordnung (EG) Nr. 1466/97 des Rates über den Ausbau der haushaltspolitischen Überwachung und der Überwachung und Koordinierung der Wirtschaftspolitiken; Verordnung (EU) Nr. 1176/2011 des Europäischen Parlaments und des Rates vom 16. November 2011 über die Vermeidung und Korrektur makroökonomischer Ungleichgewichte; Verordnung (EU) Nr. 1177/2011 des Rates vom 8. November 2011 zur Änderung der Verordnung (EG) Nr. 1467/97 über die Beschleunigung und Klärung des Verfahrens bei einem übermäßigen Defizit; Richtlinie 2011/85/EU des Rates vom 8. November 2011 über die Anforderungen an die haushaltspolitischen Rahmen der Mitgliedstaaten; alle in: Amtsblatt der EU, Nr. L 306 vom 23. November 2011; und das sogenannte ,two pack‘ aus Vorschlag für eine Verordnung des Europäischen Parlaments und des Rates über gemeinsame Bestimmungen für die Überwachung und Bewertung der Übersichten über die gesamtstaatliche Haushaltsplanung und für die Gewährleistung der Korrektur übermäßiger Defizite der Mitgliedstaaten im Euro-Währungsgebiet, KOM (2011) 821; Vorschlag für eine Verordnung des Europäischen Parlaments und des Rates über den Ausbau der wirtschafts- und haushaltspolitischen Überwachung von Mitgliedstaaten, die von gravierenden Schwierigkeiten in Bezug auf ihre finanzielle Stabilität im Euro-Währungsgebiet betroffen oder bedroht sind, KOM (2011) 819.

23 Diese Vorschläge des ,six pack' und des ,two pack' lagen vor der wesentlichen Einigung der ,Van Rompuy Task Force‘ vor, die vom Europäischen Rat am 26. März 2010 zur Ausarbeitung von Vorschlägen zur Stärkung der Stabilität und Fiskaldisziplin eingesetzt worden war. Tatsächlich orientierte sich das Ergebnis der Task Force letztendlich stark an den Kommissionsvorschlägen. Der Altschuldentilgungsfonds bezieht sich auf den Vorschlag des Sachverständigenrats der Bundesregierung vom 11. November 2011. In den Debatten um das ,two pack' haben Teile des Europäischen Parlaments beispielsweise auch für die Forderung eines Altschuldentil- 
mission und Europäisches Parlament in der Vorreiterrolle. ${ }^{24}$ Auch bei der konkreten Ausgestaltung der Vertragsdetails von EFSF und ESM sowie in den künftigen Verfahren des ESM und bei der Aushandlung der makroökonomischen Anpassungsprogramme für die Programmstaaten, die Kredithilfen aus dem EFSF oder ESM erhalten, kann der Europäische Rat auf eine starke Einbeziehung der Europäischen Kommission nicht verzichten. Selbst in den Bereichen, in denen die Unionsmethode die Zuständigkeit der Gemeinschaftsinstitutionen bestreitet, kommt sie spätestens im Politikmanagement keineswegs ohne die Kapazitäten der Gemeinschaftsinstitutionen aus. Zu hoffen bleibt, dass die Europäische Kommission auf diesem Wege auch die im Rahmen des Fiskalvertrags vorgesehenen Verfahren und Strukturen letztlich in das Europäische Semester und den Stabilitäts- und Wachstumspakt integrieren kann und so der Entwicklung einer ,Gegenregierung' durch den Euro-Gipfel entgegenwirken wird. Doch auch dann bleiben die Fragen der Kontrolle und Beteiligung des Europäischen Parlaments und einer effektiven Kontrolle und Beteiligung des Deutschen Bundestags an der Politik im Rahmen des Fiskalvertrags auf der Tagesordnung.

Gerade die zahlreichen juristischen Probleme bei der Umsetzung des Fiskalvertrags in die verschiedenen nationalen Rechtsordnungen der Mitgliedstaaten zeigen die begrenzten Möglichkeiten, die europäische Integration außerhalb des Gemeinschaftsrechts weiterzuentwickeln. Mit den europäischen Verträgen wurde eine vergleichsweise effiziente Struktur supranationalen Rechts geschaffen, die kaum durch andere völkerrechtliche Konstruktionen selbst kleinerer Zusammenschlüsse übertroffen werden kann. Deswegen ist es richtig, sich darauf zu konzentrieren, das Gemeinschaftsrecht und die europäischen Verträge dort weiterzuentwickeln, wo mehr Handlungsfähigkeit, bessere und härtere Regeln, neue Strukturen und eine stärkere demokratische Legitimation notwendig sind, anstatt die Institutionen, die diese Entwicklungen vorantreiben können, zu schwächen.

\section{Fazit: „Brügge sehen... und sterben.“ - Beendet die Unionsmethode den Erfolg der europäischen Integration?}

Die Unionsmethode wird also in der Regel nicht zu einem besseren Management der EuroKrise, zu schnelleren und effizienteren Maßnahmen gegen die Krise auf europäischer Ebene oder zu einer stärkeren Legitimität der Euro-Rettungspolitik führen können. Sie droht gleichzeitig zu einer Bremse der vertieften Integration zu werden.

Zudem ist vor dem Hintergrund der Entwicklungen in der Krise auch für das altbewährte Prinzip der Gemeinschaftsmethode die Frage nach strukturellen, organisatorischen und vertragsrechtlichen Veränderungen zu stellen. Dabei ist auch die Frage nach der künftigen Rolle des Rates der Europäischen Union und der Mitgliedstaaten im Institutionengefüge eine entscheidende, bei der Merkels Ansatz aus der Rede in Brügge das „Lagerdenken [zu] überwinden“ ${ }^{\circ 5}$ auch in Betracht gezogen werden muss. Die neuen Instrumente der Wirtschaftsregierung werden nicht einfach von einer noch so effizienten Wirtschaftsregierung allein aus der Europäischen Kommission heraus getragen sein können. Eine verbesserte Einbindung

gungsfonds gestritten. Vgl. Sachverständigenrat zur Begutachtung der gesamtwirtschaftlichen Entwicklung: Verantwortung für Europa wahrnehmen. Jahresgutachten 2011/12, Wiesbaden 2011, S. 4-10; Berichtsentwurf PA 1/2012/136.

24 Vgl. Europäisches Parlament: Plenarsitzung 13.-16. Februar: Arbeitslosigkeit, Lebensmittelhilfen und Syrien. Hintergrund zur Eurobonds-Debatte: Wider die Schuldenspirale, abrufbar unter: http://www.europarl.europa.eu/news/de/headlines/content/20120203FCS37185/8/html/Hintergrund-zur-Eurobonds-Debatte-Wider-dieSchuldenspirale (letzter Zugriff: 29.6.2012).

25 Rede von Bundeskanzlerin Merkel, 2010, S. 6. 
des Europäischen Parlaments wird ebenso notwendig sein, wie dem Rat der Europäischen Union eine wichtige Rolle zu geben. Schließlich stellen sich die Anforderungen der wirtschaftspolitischen Steuerung zuerst an die Umsetzung durch die nationalen Regierungen und die sie tragenden politischen Mehrheiten. Eine ,Gretchenfrage' der weiteren Integration wird auch sein: Welche Rolle soll der Rat der Europäischen Union spielen? Die Verfahren auf europäischer Ebene müssen zudem mit den politischen Prozessen und Debatten in den Mitgliedstaaten verzahnt werden. Die wirtschaftspolitische Steuerung darf nicht einfach ,vom Brüsseler Himmel' fallen, sondern muss neben dem Europäischen Parlament die nationalen Parlamente von Anfang an in die Politikentwicklungsprozesse einbeziehen und so zur Nachvollziehbarkeit in der Öffentlichkeit beitragen. Und auch weiterhin müssen nationale Mehrheiten unterschiedliche Wege und Modelle von Wirtschafts- und Finanzpolitik betreiben können, um Vielfalt und Fortentwicklung von Politiken zu gewährleisten.

Grundsätzlich ist trotz des Anspruches der Erklärung von Laeken zur Zukunft der Europäischen Union ${ }^{26}$ festzustellen: die europäische Integration wurde auch durch den Vertrag von Lissabon nicht genug weiterentwickelt, um die Europäische Union für die Herausforderungen der Globalisierung und gegen die Gefahr von nicht koordinierten nationalen Politiken zu wappnen. Zudem müssen neben Haushalts-, Finanz-, Wirtschafts-, Sozial- und Steuerpolitik auch Demokratie und Subsidiarität auf die Tagesordnung für zukünftige Integrationsschritte kommen. Die Institutionen müssen sich aus eigenem Antrieb der Aufgabe der Schaffung stärkerer öffentlicher Aufmerksamkeit für die Europapolitik stellen: Europäisches Parlament und Europäische Kommission müssen sich verändern, um ihre Arbeit mehr in direktem Kontakt zu den Menschen zu bringen. Das gilt auch für die Ausgestaltung der notwendigen Integrationsschritte: Nur mit einem Konvent unter Beteiligung von Zivilgesellschaft und Sozialpartnern werden wir in der Debatte über die Zukunft der Europäischen Union das Vorurteil der ,Brüsseler Hinterzimmer' ausräumen. Der Weg über die Methode Brügge hingegen könnte für die Europäische Union - wie im Film der Aufenthalt in Brügge für manchen Protagonisten - tödlich sein.

26 Erklärung von Laeken zur Zukunft der Europäischen Union, in: Europäischer Rat: Schlussfolgerungen des Vorsitzes. Europäischer Rat (Laeken) 14. und 15. Dezember 2001, SN 300/1/01, S. 19-26. 